\title{
Tidal friction in close-in planets
}

\author{
Adrián Rodríguez ${ }^{1}$, Sylvio Ferraz-Mello ${ }^{1}$ and Hauke Hussmann ${ }^{2}$ \\ ${ }^{1}$ Instituto de Astronomia, Geofísica e Ciências Atmosféricas, University of São Paulo \\ Rua do Matão, 1226, CEP 05508-900, São Paulo, Brasil \\ email: sylvio@astro.iag.usp.br, adrian@astro.iag.usp.br \\ ${ }^{2}$ Institut für Planetenforschung, DLR, Berlim-Adlershof, Germany \\ email: hauke.hussmann@dlr.de
}

\begin{abstract}
We use Darwin's theory (Darwin, 1880) to derive the main results on the orbital and rotational evolution of a close-in companion (exoplanet or planetary satellite) due to tidal friction. The given results do not depend on any assumption linking the tidal lags to the frequencies of the corresponding tide harmonics (except that equal frequency harmonics are assumed to span equal lags). Emphasis is given to the study of the synchronization of the planetary rotation in the two possible final states for a non-zero eccentricity : (1) the super-synchronous stationary rotation resulting from the vanishing of the average tidal torque; (2) the capture into a 1:1 spin-orbit resonance (true synchronization), which is only possible if an additional torque exists acting in opposition to the tidal torque. Results are given under the assumption that this additional torque is produced by a non-tidal permanent equatorial asymmetry of the planet. The indirect tidal effects and some non-tidal effects due to that asymmetry are considered. For sake of comparison with other works, the results obtained when tidal lags are assumed proportional to the corresponding tidal wave frequencies are also given.
\end{abstract}

Keywords. Tidal friction, Close-in exoplanets, Synchronization, Stationary rotation, Energy dissipation, Hot Jupiters

\section{Introduction}

It is well-known that tidal friction tends to synchronize the rotation period of a closein companion (exoplanet or planetary satellite) with the period of its orbital motion around the primary. However, it is not always taken into account the fact that there are two possible final states: (1) the super-synchronous stationary rotation resulting from the vanishing of the average tidal torque (Goldreich, 1966); (2) the capture into a spin-orbit resonance (generally the synchronous one) as a result of the vanishing of the average sum of two torques, the tidal torque and an additional torque acting in opposition to it. The selection of one or another of the two final states is determined by the orbit eccentricity and by the existence or not of a permanent non-tidal equatorial asymmetry of the close-in planet. In a 'cold' solid quasi-elastic body, the existence of one important equatorial asymmetry is not superseded by the tidal deformations and the final state is the capture into a spin-orbit resonance which may be $1: 1$, as in the case of the Moon, or a different one, as in the case of Mercury (captured into a 3:2 spin-orbit resonance; see Lemaitre et al., 2006). Otherwise, the tidal torque dominates and the final state is nonsynchronous, at least while the orbit is not circularized. This is expected to be the case for hot Jupiters, since the giant planets in our Solar System do not show any measurable equatorial asymmetry. In this case, one classical result is that the system tends to a final state whose rotation speed is given by $\Omega_{\mathrm{stat}}=n\left(1+\kappa e^{2}\right)$ where $n$ and $e$ are the orbital mean motion and eccentricity and $\kappa$ is a positive constant whose actual value depends on 
the hypotheses done on the tidal phase lag. When it is assumed that each harmonic of the tide has a phase lag proportional to its frequency, we obtain $\kappa=6$ (see Eq. 2.4); when the lag is assumed to be constant and frequency independent (MacDonald, 1964), the value corresponding to the vanishing of the average torque is $\kappa=9.5$ (Goldreich, 1966). If, however, we just assume that equal frequencies give rise to equal lags, without assuming any particular law relating lags to frequencies, the result is expressed in a different way: $\varepsilon_{0}=12 e^{2} \varepsilon_{2}$ where $\varepsilon_{0}$ and $\varepsilon_{2}$ are the phase lags of the tide harmonics whose frequencies are $2 \Omega-2 n$ and $2 \Omega-n$ respectively ( $\Omega$ is the planet's angular velocity of rotation).

\section{Planetary Rotation}

To study the planet rotation, we have to consider the torque $\mathcal{M}$ resulting from the misalignment of the tidal bulge created by the delayed response of the planet to the tide raising forces and use the angular momentum conservation. Initially, it is enough to consider only the interaction between the orbit and the tidally deformed planet: the change $\dot{\mathcal{L}}$ of angular momentum in the orbit may be compensated by a change $\dot{\mathcal{L}}_{\text {rot }}$ in the angular momentum acting on the planet in the opposite direction. Hence $\dot{\mathcal{L}}_{\text {rot }}=-\mathcal{M}$. The study of this equation is simplified by the fact that the torque $\mathcal{M}$ is, in this case, normal to the line of nodes $\left(M_{x}=0\right)$ thus allowing this equation to be decomposed into two parts which may be studied separately. We remind that the rotational angular momentum of the planet is given by $\mathcal{L}_{\text {rot }} \simeq C \Omega \mathbf{k}$ where $C$ is the moment of inertia of the planet with respect to the rotation axis and $\mathbf{k}$ is a unit vector along that axis. We obtain $C \dot{\Omega}=-M_{z}$ and $C \Omega \dot{J}=-M_{y}$, where $M_{y}, M_{z}$ are the components of $\mathcal{M}$ and $\dot{J}$ is a variation in the obliquity of the planet. Computing the tidal forces and torques and averaging the torque over the orbital period, we obtain

$$
<\dot{\Omega}>=-\frac{3 k_{d} G M^{2} R^{5}}{2 C a^{6}}\left[\varepsilon_{0}-\xi\left(e^{2}, S^{2}\right)\right]
$$

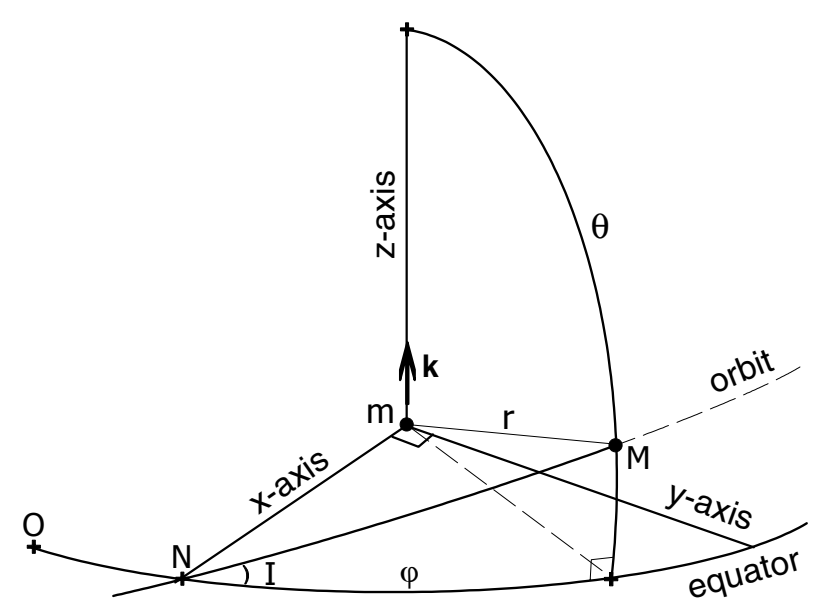

Figure 1. Spherical coordinates and reference system 
where $k_{d}$ is the dynamical Love number, $G$ the gravitation constant, $M$ the mass of the tide raising body (the star), $R$ the mean radius of the planet, $a$ the orbit semi-major axis and $\xi\left(e^{2}, S^{2}\right)$ a function whose terms are proportional to $e^{2}$ and $S^{2}$ ( $S$ is the sine of the obliquity) whose coefficients depend on the lags of the several harmonics in which the tide is decomposed. (For details, see Ferraz-Mello et al., 2007). Near synchronization,

$$
\xi\left(e^{2}, S^{2}\right)=e^{2}\left(5 \varepsilon_{0}+12 \varepsilon_{2}\right)+S^{2} \varepsilon_{0} .
$$

If we assume that the planet has no other significant deformation besides the tidal one, Eqn. (2.1) shows that, in the first approximation, the averaged acceleration $\langle\dot{\Omega}\rangle$ is proportional to $\left|\varepsilon_{0}\right|$ with a sign contrary to $\Omega-n$, indicating that the system will evolve towards synchronization.

We say that the system reaches a state of stationary rotation when the average angular acceleration (or the average tidal torque component $\left\langle M_{z}\right\rangle$ ) vanishes. Solving the equation $<\dot{\Omega}>=0$, we obtain, to the second order in $e, S$,

$$
\varepsilon_{0}=12 e^{2} \varepsilon_{2} .
$$

In Darwin's theory, as well as in many theories inspired by it, the tidal lags $\varepsilon_{0}$ and $\varepsilon_{2}$ are assumed to be proportional to the frequencies of the corresponding tide harmonics $(2 \Omega-2 n$ and $2 \Omega-n$, respectively). With this assumption, the above result becomes

$$
\Omega_{\text {stat }}=n\left(1+6 e^{2}\right)
$$

(see eqn. 6.1), which means that, when $e \neq 0$, the rotation stabilizes at a slightly supersynchronous value.

The second equation resulting from the angular momentum conservation gives the variation of the planet's obliquity:

$$
<\dot{J}>=-\frac{3 k_{d} S G M^{2} R^{5}}{2 C \Omega a^{6}} \varepsilon_{2} .
$$

The part of the equation corresponding to the vanishing of the torque along the nodal line indicates that no precession of the nodes occurs due to tidal friction.

\section{Spin-orbit synchronization}

The spin-orbit synchronization condition is given by $\Omega=n$ or, if we assume the lags proportional to the frequencies of the corresponding harmonics, $\varepsilon_{0}=0$, in which case eqns. (2.1) and (2.2) give

$$
<\dot{\Omega}>=\frac{18 k_{d} G M^{2} R^{5}}{C a^{6}} e^{2} \varepsilon_{2}
$$

which cannot vanish if $e \neq 0$. It is worth noting that $S$ does not affect the above condition (at least when terms of the fourth order in $e, S$ are neglected).

\subsection{Synchronous Asymmetric Planets}

The previous conclusions were derived from the assumption that the planet has no other significant deformation besides the tidal one. If, for instance, it has a permanent non-tidal (solid-like) equatorial ellipticity, different results follow. Indeed, in such case, we have to 
add to the potential due to the tide, the non-tidal quadrupolar term associated with the equatorial ellipticity:

$$
U_{22}=\frac{G m R^{2}}{r^{3}} J_{22} P_{22}(\cos \theta) \cos 2\left(\varphi-\varphi_{22}\right)
$$

where $m$ is the mass of the planet, $r, \theta, \varphi$ are the radius vector, co-latitude and longitude of the star in the planetocentric frame (see Fig. 1) and $P_{22}$ is an associated Legendre function. $J_{22}$ and $\varphi_{22}$ are the two parameters characterizing the ellipticity of the equator (see Beutler, 2005). If $J_{22}$ is large enough, the torque due to $U_{22}$ will be dominant and the synchronization will end with capture into an exact 1:1 spin-orbit resonance (Goldreich, 1966). In this case, we have, instead of eqn. (2.3),

$$
\varepsilon_{0}=0 \text {, }
$$

and the equations obtained in the previous section cannot be used since the torque forcing the motion to be synchronous must also be considered. Since the tidal friction in a synchronous companion moving in an eccentric orbit tends to accelerate its motion, the body will rotate faster, but, being asymmetric, the advance with respect to the symmetric configuration will create a torque in the contrary direction, which will compensate the tidal torque. The equilibrium is obtained with the body displaced of an angle $\delta$ forward. The average torque due to this displacement is

$$
<\left(M_{22}\right)_{z}>=\frac{6 G M m R^{2} J_{22} \sin 2 \delta}{a^{3}}\left(1-\frac{5}{2} e^{2}-\frac{1}{2} S^{2}\right) .
$$

The condition $\langle\dot{\Omega}\rangle=0$ allows the offset angle $\delta$ to be determined. At the order of approximation adopted in this paper,

$$
\sin 2 \delta \simeq \frac{3 M R^{3}}{m J_{22} a^{3}} k_{d} \varepsilon_{2} e^{2}
$$

This equation shows that when $J_{22}$ is too small, the right-hand side is large and the synchronization cannot be reached $(\delta$ becomes larger than the critical limit allowing synchronization) $\dagger$. Nevertheless, the concomitant process of circularization of the orbit (see Sec. 5) will continue and, at some moment in the evolution, $e$ will become enough small to allow capture into a 1:1 spin-orbit resonance.

The torque due to $U_{22}$ also contributes with a precession of the equatorial plane of the synchronous companion. However, at the order of approximation adopted here, the only effects do not depend on any misalignement $\delta \neq 0$ and are thus not of tidal origin. They are due to the figure of the companion captured into a 1:1 spin-orbit resonance.

\section{Work done by the tidal force and energy release}

The work done by the tidal forces in a displacement $d \mathbf{s}$ is given by $d W=\mathbf{F} \cdot d \mathbf{s}$, or $\dot{W}=\mathbf{F} \cdot \mathbf{v}$ where $\mathbf{v}$ is the velocity vector. In the case of close-in planets in stationary rotation, the introduction of the condition given by eqn. (2.3) gives

$$
<\dot{W}>=-\frac{3 n k_{d} G M^{2} R^{5}}{2 a^{6}}\left(7 e^{2}+S^{2}\right) \varepsilon_{2} .
$$

It is worth emphasizing that this result does not depend on any hypothesis linking lags to frequencies (the only assumptions are that equal frequencies lead to equal lags).

$\dagger$ When the tidal phase lag is assumed to be frequency independent (MacDonald theory), the resulting critical value of $J_{22}$ is proportional to $e^{4}$ (Goldreich, 1966) instead of $e^{2}$. 
If the same calculations were done using $\varepsilon_{0}=0$, instead of the condition given by eqn (2.3), we would obtain for the work the value

$$
-\frac{3 n k_{d} G M^{2} R^{5}}{2 a^{6}}\left(19 e^{2}+S^{2}\right) \varepsilon_{2} .
$$

However, the latest value is not the net work done because a synchronous rotation cannot exist without additional forces acting in the system when $e \neq 0$. As the additional forces act in opposition to the tidal forces, the work done by them has a different sign. From (3.2), we indeed obtain

$$
<\dot{W}_{22}>=\frac{6 G M m R^{2} n J_{22} \sin 2 \delta}{a^{3}}\left(1-\frac{5}{2} e^{2}-\frac{1}{2} S^{2}\right) \simeq \frac{18 n k_{d} G M^{2} R^{5}}{a^{6}} e^{2} \varepsilon_{2},
$$

where the rightmost expression was obtained introducing the value of $\sin 2 \delta$ given by eqn. $(3.5)$.

When this term is added to the direct tidal work given by eqn. (4.2), the result becomes equal to that given by eqn. (4.1). This is the same result found by several authors (Segatz et al. 1988; Levrard, 2008; Wisdom, 2008) and may also be obtained from the results of Peale and Cassen (1978) on energy dissipation due to tidal friction. In the comparisons, the phase lag $\varepsilon_{2}$ of the tide harmonic whose frequency is $2 \Omega-n$, is related to the planet's quality factor through $Q=1 / \varepsilon_{2}$.

\subsection{Energy Dissipation}

The total energy variation of the system must be equal to zero. Besides the orbital energy, whose variation is given above, we have the rotational energy of the deformed body and the thermal energy dissipated in the body. This balance equation allows us to calculate the energy dissipated in the planet due to the tides raised on it by the star.

The variation of the rotational energy is given by $C \Omega \dot{\Omega}$ and vanishes when $\dot{\Omega}=0$. Therefore, in the studied cases, $|\dot{W}|$ may be roughly equal to the thermal energy released inside the companion (they are equal when we neglect the change in the equilibrium rotation speed due to the variation of the mean motion, which is of the order of $(R / a)^{2}$.)

\section{Variation in the orbital elements}

The variation of the mean motion, semi-major axis, eccentricity and obliquity can be known from the variations of the energy and angular momentum (see Ferraz-Mello et al., 2007) using classical two-body dynamics equations. For the mean-motion, we obtain

$$
<\dot{n}>=-\frac{3 n}{2 a}<\dot{a}>=-\frac{3 n a}{G M m}<\dot{W}>
$$

or

$$
<\dot{n}>=\frac{9 n^{2} k_{d} M R^{5}}{2 m a^{5}}\left(7 e^{2}+S^{2}\right) \varepsilon_{2} .
$$

We note that in some papers (e.g. Mardling and Lin, 2004), the term $19 e^{2}$ appears in the equation giving $\langle\dot{n}\rangle$ instead of $7 e^{2}$. That result is only partial because it does not take into account that a torque counteracting the tidal torque is necessary to allow the synchronization to be achieved. 
For the eccentricity, we obtain

$$
<\dot{e}>=-\frac{21 n e k_{d} M R^{5}}{2 m a^{5}} \varepsilon_{2} .
$$

At the order considered here, the torque due to $U_{22}$ does not contribute to the variation of the eccentricity. Its only contribution is a motion of the node whose secular part is

$$
<\dot{\widehat{O N}}>=\frac{3 J_{22} n R^{2}}{a^{2}}\left(1-2 e^{2}-\frac{1}{4} S^{2}\right) \cos 2 \delta
$$

However, this precession exists even when $\delta=0$ and, thus, it is not an effect due to the tidal misalignment of $U_{22}$, but an effect due to the trapping of the planet into the $1: 1$ spin-orbit resonance.

The obliquity is the angle between the equator and the orbital plane and we have to consider the possible variation of both planes. The torque of the forces acting on the main body gives the variation of the orbital plane with respect to a fixed frame; its reaction, acting on the companion gives the variation of the equatorial plane. If we call $I$ the angle fixing the orbital plane and $J$ the angle fixing the equator (w.r.t. an inertial plane), we obtain, for close-in synchronous or near-synchronous planets: $\langle\dot{I}\rangle /\langle\dot{J}\rangle$ $\propto(R / a)^{2} \ll 1$. This means that we may neglect the variation of the obliquity due to the motion of the orbital plane when compared to the variation due to the motion of the equator.

\section{Linear theories with a constant time lag}

For the sake of comparison with theories where the phase lags $\varepsilon_{i}$ are assumed to be proportional to the frequencies of the corresponding tide harmonics, with $\tau$ (time lag) as the coefficient of proportionality, we give below the resulting equations.

For the parameters related to the rotation of the close-in companion we have

$$
\begin{gathered}
<\dot{\Omega}>=\frac{3 n k_{d} G M^{2} R^{5}}{C a^{6}}\left[\left(1+\frac{27}{2} e^{2}-\frac{1}{2} S^{2}\right)-\left(1+\frac{15}{2} e^{2}-\frac{1}{2} S^{2}\right) \frac{\Omega}{n}\right] \tau \\
<\dot{J}>=-\frac{3 n k_{d} G M^{2} R^{5}}{C \Omega a^{6}} S\left(1-\frac{1}{2} \frac{\Omega}{n}\right) \tau .
\end{gathered}
$$

For the equations giving the variation of the orbital elements, we have

$$
\begin{gathered}
<\dot{n}>=-\frac{3 n}{2 a}<\dot{a}>=\frac{9 n^{3} k_{d} M R^{5}}{m a^{5}}\left[\left(1+23 e^{2}\right)-\left(1+\frac{27}{2} e^{2}-\frac{1}{2} S^{2}\right) \frac{\Omega}{n}\right] \tau, \\
<\dot{e}>=-\frac{27 n^{2} e k_{d} M R^{5}}{m a^{5}}\left(1-\frac{11}{18} \frac{\Omega}{n}\right) \tau,
\end{gathered}
$$

These equations are the same given by Hut (1981) and several papers on tidal friction on close-in exoplanets using Hut's approach (e.g. Mardling and Lin, 2004; Dobbs-Dixon et al., 2004). It is worth noting that the equations in the cited papers are expanded to a much higher order in eccentricity and that the quality factor adopted there is related to the time lag used here through $Q=1 / n \tau$.

To obtain the equations corresponding to the free stationary rotation, it is enough to replace $\Omega / n$ by $\left(1+6 e^{2}\right)$. 
Sometimes, the condition $\Omega=n$ is introduced to obtain the equations corresponding to the case of a planet locked in a 1:1 spin-orbit resonance. However, the results thus obtained are inconsistent. For instance, eqn. (6.1) gives, in such case,

$$
<\dot{\Omega}>=\frac{18 n k_{d} G M^{2} R^{5}}{C a^{6}} e^{2} \tau
$$

that is, a non-zero acceleration (when $e \neq 0$ ) showing that the condition $\Omega=n$ cannot subsist. The results, obtained with such condition are only partial and to become correct need that the contributions coming from $M_{22}$ (or from another forcing torque) be added to the right-hand sides.

\section{Conclusion}

This communication presents some consequences of Darwin's theory for bodily tides. As expected, the main results formally coincide with the general results given by Kaula (1964) and Hut (1981) to the order of approximation adopted here, when we do the same hypotheses on the physics of the lagging done by them. Emphasis is given in the paper to companions having reached one of the two possible end states of the planet synchronization. If tides are the only source of perturbations in the system, the perfect synchronization cannot be achieved while the orbit is not completely circularized. If a remnant eccentricity exists, the final state is rather non-synchronous and the rotation reaches a stationary state with a rotation velocity slightly larger than the orbital meanmotion. In order to have a true synchronous rotation, it is necessary to provide the system with a torque counteracting the tidal torque. This additional torque can, for instance, be due to a permanent non-tidal solid-like equatorial asymmetry of the companion. Tides drive the figure of the body to a small misalignment which creates a torque acting in opposition to the tidal torque. At the order considered here, the results for the tidal variations of the orbital elements are the same in the stationary (free) and synchronous (forced) cases but, in the synchronous case, we have some additional non-tidal effects coming from the figure of the body captured into a 1:1 spin-orbit resonance, which also affects the evolution of the system and may be considered.

\section{Acknowledgements}

The authors acknowledge the support of CNPq, DFG, FAPESPand the CAPES/SECYT cooperation programme. We thank the referee for his clever comments.

\section{References}

Darwin, G. H., 1880, Philos. Trans. 171, 713 (repr. Scientific Papers, Cambridge, Vol. II, 1908). Dobbs-Dixon, I., Lin, D. N. C. and Mardling, R. A. 2004, Astrophys. J. 610, 464

Ferraz-Mello, S., Rodríguez, A. \& Hussmann, H., 2007, Cel. Mech. Dynam. Astron. (submitted). ArXiv: astro-ph 0712.1156

Goldreich, P. 1966, Astron. J. 71, 1

Hut, P., 1981, Astron. Astrophys. 99, 126

Kaula, W. M. 1964, Rev. Geophys. 3, 661

Lemaitre, A., D’Hoedt, S., \& Rambaux, N., 2006, Cel. Mech. Dynam. Astron. 95, 213

Levrard, B., 2008, Icarus (in press)

MacDonald, G. F., 1964, Rev. Geophys. 2, 467.

Mardling, R. A. \& Lin, D. N. C., 2004 Astrophys. J. 614, 955 
Peale, S. J. \& Cassen, P., 1978, Icarus, 36, 245-269.

Segatz, M., Spohn, T., Ross, M. N., \& Schubert, G., 1988, Icarus, 75, 187-206.

Wisdom, J., 2008, Icarus (in press). 